\title{
Anestesia neuroaxial en cirugía ambulatoria hoy: revisión de la literatura
}

Neuraxial anesthesia in outpatient surgery today: review of the literature

https://doi.org/10.25237/carsach2020.05

Dra. Francisca Cabrera V. ${ }^{1}$

${ }^{1}$ Servicio de Anestesiología del Hospital del Trabajador de la Asociación Chilena de Seguridad.

Profesor asociado Facultad de Medicina de la Universidad Andrés Bello.

\section{Autor Corresponsal:}

Dra. Francisca Cabrera V.

Servicio de Anestesiología del Hospital del Trabajador de la Asociación Chilena de Seguridad.

Dirección: Ramón Carnicer 185, Providencia, Santiago.

Teléfono: 226853333

E-mail: fcabrera@achs.cl

ORCID ID: https://orcid.org/0000-0002-5597-6698

Palabras clave: anestesia neuroaxial, cirugía ambulatoria, anestesia, espinal, peridural, ambulatorio.

Key words: neuraxial anesthesia, ambulatory surgery, spinal, anesthesia, epidural, outpatient

\section{Puntos clave}

- La anestesia neuroaxial es aplicable en el contexto de cirugía ambulatoria dado que puede ser efectiva y eficaz.

- Las técnicas neuroaxiales presentan diversas ventajas en relación a la anestesia general, siendo de particular relevancia en estos tiempos la disminución del riesgo de contagio de Covid-19, al evitar la manipulación de la vía aérea.

- El anestesiólogo debe conocer los anestésicos locales disponibles para así privilegiar el uso de los de duración corta o aplicar la evidencia existente para optimizar el uso de dosis bajas logrando una buena calidad anestésica.

- Al implementar protocolos, podemos optimizar el uso de la anestesia neuroaxial ambulatoria y a su vez disminuir las complicaciones como la retención urinaria.

\section{Resumen}

La anestesia neuroaxial es una alternativa aplicable a la cirugía ambulatoria, pero para poder tener éxito y eficiencia, debemos conocer las bases y manejar las variables para optimizar su uso. Las técnicas neuroaxiales nos ofrecen ventajas clínicas que pueden favorecer a nuestros pacientes, y en los tiempos actuales que vivimos, podemos disminuir el riesgo de transmisión de COVID-19 al personal de salud. Para implementar la anestesia espinal en un contexto ambulatorio, debemos crear protocolos para estandarizar la técnica teniendo en consideración el uso de anestésicos locales de duración corta, dosis bajas y evitar complicaciones como la retención urinaria. Al estandarizar, lograremos obtener los beneficios de la técnica y a su vez ser eficientes teniendo tiempos de alta adecuados con un mínimo de efectos adversos. 


\begin{abstract}
Neuraxial anesthesia is an alternative applicable to outpatient surgery, but in order to be successful and efficient, we must know the basics and manage the variables to optimize its use. Neuraxial techniques offer us clinical advantages that can benefit our patients, and in the current times we live in, reduce the risk of Covid-19 transmission to health care workers. To implement spinal anesthesia in an outpatient setting, we must create protocols to standardize the technique, taking into account the use of short-duration, low-dose local anesthetics and avoiding complications such as urinary retention. By standardizing, we will be able to obtain the benefits of the technique and at the same time be efficient, having adequate discharge times with a minimum of adverse effects.
\end{abstract}

\title{
Introducción
}

La anestesia ideal para la cirugía ambulatoria debe caracterizarse por seguridad, eficacia y confiabilidad. Es deseable que sea de administración rápida y que exista un control preciso tanto de su inicio como de su fin. Otros factores muy importantes a considerar son que tenga mínimos efectos adversos, que permita el alta precoz, y que tenga un grado alto de satisfacción del paciente con respecto a la técnica (1).

La anestesia neuroaxial es una técnica que puede cumplir todas las características antes mencionadas. Como anestesiólogos debemos considerar estas variables y aplicar conceptos para optimizar su utilización en el contexto de cirugía ambulatoria.

El objetivo de esta revisión es entregar conceptos básicos de la anestesia espinal ambulatoria, las ventajas que podemos obtener de esta técnica, cómo optimizar su uso y evitar complicaciones. Además, se evaluará algunos aspectos importantes de la anestesia peridural ambulatoria.

\section{Metodología de revisión de literatura}

Para esta revisión, se realizó una búsqueda bibliográfica en Medline a través de PubMed de artículos publicados desde el año 1990 a la fecha. Los términos de búsqueda fueron neuraxial anesthesia, spinal anesthesia, epidural anesthesia, outpatient, ambulatory. Además, para los subtemas tratados se hizo una búsqueda dirigida, incluyendo términos como Covid-19 and spinal anesthesia, unilateral spinal anesthesia, urinary retention and spinal anesthesia y postdural puncture headache.

\section{Anestesia espinal ambulatoria}

En el contexto ambulatorio, la utilización de anestésicos locales de duración corta ha demostrado tener un perfil favorable de efectos adversos y tiempos de alta al compararlo con anestesia general (2). A su vez, su uso espinal se ha asociado con menores cifras de dolor y requerimientos analgésicos en la unidad de recuperación anestésica (3).

Al revisar estudios que comparan anestesia espinal con anestesia general ambulatoria, el meta-análisis publicado por Liu et al el año 2005 (3), evidenció que la anestesia neuroaxial presenta mayor tiempo de inducción, pero con menores cifras de dolor y requerimientos de analgésicos en la unidad de recuperación. Por otro lado, la anestesia espinal presenta mayor tiempo de estadía en recuperación y mayor tiempo en ser dados de alta. Sin embargo, hay que considerar que es un meta-análisis con alta heterogeneidad, las dosis y tipos de anestésicos locales fueron variadas (bupivacaína, lidocaína y procaína) y que aún no se utilizaban los nuevos anestésicos locales de duración corta.

En un estudio prospectivo observacional publicado por Capdevilla et al (4), se buscó determinar cuáles eran los factores que influían en la elección de la técnica anestésica en una cohorte de pacientes ambulatorios. Los pacientes en su mayoría fueron sometidos a procedimientos ortopédicos y urológicos, siendo la decisión del paciente el principal factor que influyó en la elección de la técnica. Sin embargo, es interesante analizar los distintos outcomes que utilizaron para comparar ambas técnicas. Los pacientes que recibieron anestesia general, orinaron más rápido (34 minutos), deambularon antes (25 minutos) y recibieron analgésicos de rescate antes (68 minutos), pero no hubo diferencias en los tiempos de alta al compararla con anestesia espinal. El dolor fue más frecuente en el grupo de anestesia general $(9,2 \%$ versus $3,6 \%)$. Los tiempos hasta la deambulación y alta, fueron más cortos en los pacientes que recibieron anestesia espinal con cloroprocaína versus otros anestésicos locales de duración más larga. Esto evidencia 
que la anestesia espinal es una alternativa válida para la cirugía ambulatoria, sobre todo si utilizamos anestésicos de duración corta y optimizamos la técnica.

\section{¿Qué ventajas tiene la anestesia espinal?}

- Control del dolor agudo, con menores requerimientos de opioides o analgésicos de rescate en el postoperatorio. Además, reduce los efectos fisiológicos producidos por el estrés quirúrgico, potenciando otros beneficios de la anestesia regional (5).

- Reducción de náuseas y vómitos postoperatorios (NVPO), al evitar la anestesia general y/o reducir e consumo de opioides en el postoperatorio (6). Esto puede ser un factor determinante en el contexto de cirugía ambulatoria. Sin embargo, hay que tener en consideración que la anestesia espinal puede producir NVPO por otros mecanismos como hipotensión, uso de opioides intratecales o bloqueo simpático que resulta en hiperactividad gastrointestinal mediado por el sistema parasimpático (5).

- Aún no hay evidencia clara que la anestesia regional disminuya el riesgo de disfunción cognitiva postoperatoria al compararla con anestesia general (7). Sin embargo, realizar un análisis de los estudios es complejo dada la heterogeneidad de evaluaciones, definiciones, etc., por lo tanto, aún es una materia a continuar investigando.

- La anestesia neuroaxial ha demostrado ser beneficiosa en pacientes con enfermedad pulmonar preexistente (8) además de reducir las complicaciones asociadas a la anestesia general. En este mismo ámbito, ha demostrado reducción de complicaciones en pacientes con apnea obstructiva del sueño (9).

En la era actual de la pandemia por Covid-19, la anestesia espinal puede ser una buena alternativa para prevenir la transmisión del virus debido a la manipulación de la vía aérea en la anestesia general. Si bien aún no existen datos exactos de la transmisión del virus de paciente al personal de salud, los datos en relación al brote de SARS-CoV del año 2003 indican que los procedimientos que generan aerosoles aumentan el riesgo de contraer la enfermedad hasta 6 veces (10). También hay que considerar, que al evitar la anestesia general, podemos prevenir náuseas, vómitos y tos en la unidad de recuperación, que también pudiese poner en riesgo al personal de salud que ahí trabaja (11).

\section{¿Cómo podemos optimizar técnica de la anestesia espinal?}

\section{Elegir el agente adecuado}

La selección del anestésico local adecuado es de vital importancia para el éxito de una anestesia espinal ambulatoria.

Es necesario el uso de anestésicos locales de duración corta y predecible, que permitan dar una anestesia de buena calidad y a su vez que el paciente pueda irse de alta en un rango de tiempo razonable y acorde al flujo de una unidad de cirugía ambulatoria.

La lidocaína ha sido ampliamente utilizada para anestesia espinal ambulatoria, con buenos resultados clínicos, sin embargo, su uso ha disminuido debido a la alta incidencia de síntomas neurológicos transitorios (SNT). En una revisión sistemática, se observó un RR de 4,35 de presentar SNT en anestesias espinales con lidocaína, al compararlo con bupivacaína, mepivacaína, prilocaína o procaína (12). Además, se observó que no se asociaba a patología neurológica previa y en todos los pacientes los síntomas desaparecieron al décimo día postoperatorio.

Bupivacaína puede ser una buena alternativa en nuestra realidad nacional. Sin embargo, en pacientes ambulatorios, la bupivacaína espinal tiene el riesgo de producir un bloqueo motor prolongado que demore el alta de los pacientes, para lo cual debemos diseñar protocolos que optimicen su uso. No sólo debemos conocer los medicamentos y las dosis a utilizar, sino también nuestra realidad asistencial y tiempos quirúrgicos, para así poder otorgar anestesia de buena calidad.

Lemoine et al (13) en una revisión sistemática de trabajos utilizando bupivacaína en anestesia espinal ambulatoria, buscaron determinar la dosis óptima para tener buenos resultados clínicos. Se excluyeron estudios con anestesia espinal unilateral. Se determinó que una dosis de bupivacaína 7,5 mg permite resolución del bloqueo motor y deambulación a los 300 minutos en un $95 \%$ de los pacientes. No se observó que la baricidad fuese un factor determinante en los outcomes. La adición de fentanyl no afectó el tiempo de deambulación, pero demoró el alta en 45 minutos. Por otro lado, las dosis de $5 \mathrm{mg}$ o inferiores se asociaron a alta tasa de falla de la técnica. 
En otra revisión sistemática de Nair et al (14), se incluyeron estudios para evaluar la anestesia espinal en cirugía artroscópica de rodilla ambulatoria. Se incluyeron 15 estudios, en su mayoría con uso de bupivacaína hiperbárica. Se evaluaron distintos outcomes:

Dosis: en anestesia espinal lateralizada, una dosis de 4-5 mg de bupivacaína hiperbárica es suficiente para dar una buena anestesia y con baja tasa de falla. Dosis mayores de 6 a 7,5 mg sólo aumenta el tiempo de recuperación, sin mejorar las tasas de falla. Al realizar una anestesia espinal no lateralizada, las dosis de $5 \mathrm{mg}$ tienen una tasa de falla de hasta $25 \%$. Dosis mayores (10 a $15 \mathrm{mg}$ ), tienen tiempos de recuperación prolongados, lo que hace que no sean recomendables para el contexto ambulatorio. En promedio, se reportaron tiempos de alta entre 180 a 240 minutos, y tiempos a la primera micción de 170 a 240 minutos.

- Coadyuvantes: todos los estudios reportaron una incidencia alta de efectos secundarios al adicionar fentanyl intratecal. El prurito se presenta en 48-75\% de los pacientes (versus 0-4\% de los que no reciben fentanyl), sin embargo sólo un 15-23\% requirió tratamiento. Otros efectos adversos como hipotensión $(<1 \%)$ y retención urinaria $(<1 \%)$ se distribuyeron igual en ambos grupos. Pese a esto, los grupos que recibieron opioides presentaron menor dolor y requerimientos de analgésicos en el postoperatorio. En definitiva, el uso de opioides intratecales aumentó el tiempo de alta en 14 minutos y aumentó la incidencia de prurito, pero tiene el beneficio de disminuir el dolor postoperatorio.

- Anestesia espinal unilateral o bilateral: la anestesia unilateral se define como el posicionamiento del paciente en decúbito lateral hacia el lado a operar por 10 a 15 minutos después de administrada la anestesia espinal. Anestesia bilateral se define como el posicionamiento en decúbito supino inmediatamente después de administrada la anestesia. Los pacientes con anestesia unilateral se recuperan más rápido y son dados de alta un promedio de 42 minutos antes que los pacientes con anestesia bilateral. La anestesia unilateral además tuvo menor incidencia de efectos secundarios (hipotensión, bradicardia). El grupo de anestesia unilateral presentó mayor retención urinaria pero no fue estadísticamente significativo.

- Complicaciones: no hubo reportes de SNT. La complicación más frecuente fue la CPPD con una incidencia entre 1 a 4\%. Otras complicaciones como NVPO o retención urinaria fluctuaron entre 0 y $1 \%$

En suma, la evidencia acumulada sugiere que la anestesia espinal con bupivacaína es una alternativa real y efectiva para la cirugía ambulatoria. Es recomendable utilizar dosis bajas y lateralizar en la medida de lo posible. El uso de coadyuvantes, como fentanyl a dosis bajas, puede ayudar en el manejo del dolor postoperatorio pero debemos tener en consideración los efectos secundarios.

\section{Nuevas alternativas de anestésicos locales de duración corta}

El uso de anestésicos locales como bupivacaína, ropivacaína o levobupivacaína, considerados de acción larga, tienen el beneficio de otorgar excelentes condiciones anestésicas intraoperatorias, pero el potencial de retraso del alta de un paciente ambulatorio (15). Reducir la dosis de estos medicamentos y adicionar coadyuvantes, puede mejorar el perfil de recuperación, pero conlleva el riesgo de falla de la técnica. Es por esto, que han surgido nuevos anestésicos locales con un perfil más favorable para el paciente ambulatorio.

2- Cloroprocaína: es un amino éster de duración corta, con un perfil farmacológico similar a la lidocaína, de latencia y duración corta pero con baja incidencia de SNT. La cloroprocaína muestra un perfil favorable para cirugía ambulatoria, mejor que lidocaína, articaína o dosis bajas de bupivacaína (16). En una revisión publicada en relación a cloroprocaína (17) se establece la factibilidad de su uso para cirugía de corta duración, incluso generando recomendaciones de dosis aproximadas según tiempo quirúrgico: $30 \mathrm{mg}$ para cirugías de 40 minutos hasta $60 \mathrm{mg}$ para las de 90 minutos. Otra ventaja, es que no hay reportes de retención urinaria en anestesias espinales con cloroprocaína (18).

\section{Este medicamento aún no está disponible en Chile para su uso intratecal.}

Prilocaína: es una amida de duración corta, y con baja incidencia de SNT. En estudios prospectivos, se ha visto una incidencia de SNT de 0,82\% (19). Las soluciones hiperbáricas han mostrado un mejor perfil para el paciente ambulatorio: menor latencia, recuperación motora más rápida y menor tiempo a la primera micción (20). Se recomienda una dosis de prilocaína hiperbárica $60 \mathrm{mg}$ para lograr un buen resultado anestésico y con buena satisfacción del paciente. En cirugías en las cuales sólo se requiere saddle block, una dosis de prilocaína hiperbárica 10 mg sería suficiente (21). Al compararla con cloroprocaína, ésta última presenta menor duración y tasa de retención urinaria (20). 
En la actualidad, los estudios existentes son pequeños, lo cual hace necesario mayor investigación para evaluar de mejor manera su uso en anestesia espinal.

Este medicamento aún no está disponible en Chile para su uso intratecal.

\section{Anestesia espinal unilateral}

Una manera de optimizar el uso de anestésicos locales intratecales podría ser la lateralización de la anestesia mediante el uso de soluciones hiperbáricas. Existen distintos estudios que intentan definir ventajas y dosis óptimas. En el estudio de Borghi et al (22) se compararon 3 dosis de bupivacaína hiperbárica 0.5\% ( 4, 6 y $8 \mathrm{mg}$ ) lateralizando a los pacientes por 15 minutos y bajo modalidad de inyección lenta $(3 \mathrm{ml} / \mathrm{min})$. La anestesia fue exitosa en el $100 \%$ de los pacientes, siendo la única diferencia significativa que en el grupo de $4 \mathrm{mg}$ no se alcanzó bloqueo motor completo de la extremidad a operar. Hubo más compromiso hemodinámico en el grupo de dosis $8 \mathrm{mg}$ y el grupo de $4 \mathrm{mg}$ cumplió condiciones de alta de manera más rápida (71 min, versus 82 y 97 minutos respectivamente). No hubo reportes de sondeo vesical. Este trabajo avala el uso de dosis bajas en anestesia espinal unilateral, lo cual puede ser beneficioso en altas más precoces, pero sobre todo en disminuir riesgo de alteraciones hemodinámicas y riesgo de retención urinaria postoperatoria.

Al comparar anestesia unilateral versus bilateral, Fanelli et al (23) utilizaron bupivacaína hiperbárica $0.5 \% 8 \mathrm{mg}$ en ambos grupos. Se demostró que en el grupo unilateral, el bloqueo era más intenso y demoraba más en regresar en la extremidad operada, la hemodinamia era más estable pero que no había diferencias en el perfil de recuperación o alta. El uso de anestesia unilateral agregaba 3 minutos al tiempo preoperatorio. El sondeo vesical fue más frecuente en el grupo bilateral, pese a que no fue estadísticamente significativo. Esto se puede deber a que se utilizó más volumen endovenoso para el manejo de la hemodinamia o a la extensión del bloqueo espinal, variables que debiésemos tener en consideración al momento de definir nuestra técnica.

En definitiva, la lateralización nos permite disminuir la dosis de anestésico local utilizado, lo que conllevaría el beneficio de altas más precoces, menor alteración hemodinámica y tendencia (no demostrada) a menor retención urinaria.

\section{¿Cómo podemos evitar complicaciones?}

\section{Retención urinaria.}

Los factores de riesgo para retención urinaria postoperatoria son edad, sexo masculino, cirugía pélvica, cirugía prolongada e historia de disfunción urológica. Los pacientes con estas características debiesen ser considerados de alto riesgo y requieren manejo dirigido independientemente de la anestesia planificada (24).

¿Qué medidas debiésemos tomar en pacientes de bajo riesgo? La micción post anestesia neuroaxial requiere la resolución del bloqueo parasimpático asociado a raíces nerviosas de S2 a S4, que generalmente son los últimos segmentos en recuperarse.

La retención urinaria postoperatoria no sólo se relaciona al bloqueo residual, sino también a la sobredistensión de la vejiga, lo cual a su vez distiende el músculo detrusor más allá de su punto funcional efectivo. Cabe además señalar que una vez que se descomprime la vejiga sobredistendida, puede persistir un grado de disfunción vesical en aquellos pacientes en que tuvieron volúmenes mayores a $600 \mathrm{ml}$ por más de 2 horas. (25).

Por lo tanto, para evitar la disfunción vesical postoperatoria debiésemos enfocarnos en 2 estrategias: control de administración de volumen endovenoso y anestésicos locales de duración corta.

En relación al volumen endovenoso, existen trabajos evidenciando que a mayor volumen administrado, mayor riesgo de distensión vesical y retención urinaria (26). Sería entonces recomendable limitar el volumen administrado en los pacientes de cirugía ambulatoria al mínimo necesario $(500 \mathrm{ml}$, máximo $1000 \mathrm{ml})$ dependiendo del procedimiento a realizarse.

Como ya se discutió previamente, es necesario restringir la dosis de anestésico local (14) para que la resolución de la anestesia espinal sea rápida y evitar la distensión de la vejiga. En relación a la baricidad o anestesia espinal unilateral, ninguna de estas estrategias ha demostrado tener un impacto importante en la incidencia de retención urinaria postop- 
eratoria (27). La adición de fentanyl como coadyuvante a lidocaína, mepivacaína y bupivacaína (27) no ha retrasado el tiempo a la primera micción post anestesia espinal.

Si aplicamos las medidas antes mencionadas, prácticamente todos los pacientes debiesen ser capaces de orinar espontáneamente después de una anestesia espinal para cirugía ambulatoria, sin mayor riesgo de retención urinaria que en una anestesia general.

Los pacientes de riesgo alto deben orinar antes del alta. Dependiendo de los protocolos locales, será el tiempo que se le otorgue para intentar orinar espontáneamente. De no lograrlo, se debe evaluar si existe sobredistensión vesical (evaluación con ultrasonido). Los pacientes que presenten volúmenes mayores a $600 \mathrm{ml}$, se benefician de sondeo vesical. Luego existen 2 caminos a seguir: dejarlos en observación hasta orinar, o darlos de alta con la instrucción de consultar si no han presentado micción luego de 6 a 8 hrs (24). Los pacientes de riesgo bajo pueden ser dados de alta con estas últimas indicaciones, y la incidencia de retención urinaria no será mayor que la de una cirugía ambulatoria bajo anestesia general (28).

\section{Cefalea post punción dural}

Existen pocos estudios que evalúen en forma dirigida la incidencia de cefalea post punción dural (CPPD) en el contexto ambulatorio. Sin embargo, es claro que el avance en el diseño de las agujas espinales y el menor calibre de éstas ha permitido que la tasa de CPPD sea entre 1 a $2 \%$ en pacientes ambulatorios y parturientas (29).

En uno de los pocos estudios prospectivos dirigidos a evaluación de incidencia en pacientes ambulatorios (30), se observó un 2\% de incidencia de CPPD en pacientes entre 15 y 45 años. En todos los pacientes se usó aguja punta lápiz número 27 gauge. Destaca que el grupo de mayor riesgo es el de 15 a 19 años, con una incidencia de 16,7\%. Otro factor para considerar es el antecedente de cefaleas, pacientes en los cuales la incidencia fue $15,4 \%$.

A nivel general, los pacientes jóvenes tienen mayor riesgo de presentar CPPD (14\% versus $7 \%$ en los mayores de 70 años) (31) dado que la duramadre de los pacientes mayores sería menos elástica y por lo tanto menos propensa a presentar una filtración de líquido cefalorraquídeo. Pacientes mujeres y parto vaginal, también tienen mayor riesgo de CPPD. Existe evidencia controvertida que los pacientes obesos mórbidos tendrían menor incidencia de CPPD (32).

Por otro lado, están los factores de riesgo de la técnica. Tradicionalmente se ha demostrado que las agujas de punta cortante (Quincke) tienen mayor incidencia de CPPD (6,6\%) al ser comparadas con las puntas cónicas de las agujas Sprotte y Whitacre (1,7\%) (33). El mayor calibre de la aguja también ha evidenciado ser un factor de riesgo, independiente de su diseño de punta (34). Otro factor de riesgo que se menciona en la literatura es la orientación de inserción de la aguja: si se inserta el bisel paralelo al eje largo de la columna, habría menor disrupción de las fibras de la duramadre al separarlas y no cortarlas. Esto favorecería el cierre del sitio de punción, impidiendo mayor filtración de líquido cefalorraquídeo (35). Sin embargo, un trabajo publicado por Reina et al (36), realizó una serie de punciones espinales en modelos in vitro con agujas 27G Whitacre, 29G Quincke paralelo al eje espinal y 29G Quincke perpendicular al eje espinal y evaluó las lesiones vía microscopio electrónico. Los resultados evidencian que, todas las punciones, produjeron lesiones pequeñas en las meninges y no había diferencia entre las distintas agujas. Tampoco hay diferencias en las lesiones al cambiar la orientación de inserción. Además, se observó que el daño de la aracnoides sería el que contribuye principalmente a las características de la CPPD, dado que la duramadre tiende a recuperarse rápidamente del daño producido por la punción. Se concluye que al usar agujas de bajo calibre, el diseño de la punta y la orientación de inserción, no serían factores de riesgo relevantes para la aparición de CPPD.

Según la evidencia presentada, no se ha demostrado mayor incidencia de CPPD en anestesia espinal ambulatoria, pero sí es recomendable hacer un enfrentamiento de prevención global de esta complicación en todos los pacientes.

\section{Síntomas neurológicos transitorios}

La aparición de síntomas neurológicos transitorios es una preocupación ante la ejecución de una anestesia espinal, y es el motivo por el cual la lidocaína ha caído en desuso para estos fines. En un estudio que evaluó outcomes de anestesia espinal realizada con bupivacaína y lidocaína en 250 pacientes (37), se observó una incidencia de 13\% en bupivacaína y 55-80\% con lidocaína hiperbárica. Cabe señalar que la mayor parte de los casos se presentó en los pacientes operados en posición de litotomía, factor de riesgo establecido para presentar SNT. No se encontró evidencia que el tipo de aguja utilizada en la anestesia espinal fuese un factor de riesgo.

En relación a los nuevos anestésicos locales de duración corta, recientemente se publicó una revisión Cochrane (38) para evaluar su incidencia de SNT. El riesgo de presentar SNT se reduce entre 82 y $90 \%$ al usar bupivacaína, lev- 
obupivacaína, prilocaína, procaína y ropivacaína en relación al uso de lidocaína. En el caso de cloroprocaína, se observó una incidencia similar a lidocaína, sin embargo, esta conclusión fue obtenida por el resultado de un solo estudio pequeño en el cual hubo casos de SNT, por lo tanto, es una materia en la cual aún se debe investigar. En todos los pacientes que presentaron SNT, éstos se resolvieron a los 5 días de evolución.

\section{¿En qué cirugías es aplicable la anestesia espinal ambulatoria?}

Si bien la mayoría de los trabajos están orientados a cirugía traumatológica o de extremidades inferiores, también existen de otros tipos como la laparoscopía. Vaghadia et al (39) publicaron un estudio en laparoscopía ginecológica de corta duración, comparando 3 grupos de distintas dosis de lidocaína y sufentanyl (20mg y 25ug, 20mg y 10ug, 10mg y 10ug). Las calidades de anestesia fueron definidas como buena o excelente en todas las pacientes, con tiempos de recuperación similares (entre 135 y 90 minutos), siendo una buena alternativa para el contexto ambulatorio.

Sin embargo, dada la alta incidencia de SNT asociada a lidocaína, se ha investigado el uso de otros anestésicos locales. De Santiago et al (40) compararon el uso de lidocaína $10 \mathrm{mg}$ asociado a fentanyl $10 \mathrm{ug}$, con levobupivacaína 3 mg más fentanyl 10 ug, en cirugía laparoscópica ginecológica ambulatoria. Se observó que el grupo con levobupivacaína era comparable al grupo lidocaína en cuanto a resolución del bloqueo, condiciones intraoperatorias y satisfacción del paciente, haciéndolo una alternativa factible.

Por lo tanto, la anestesia espinal es una alternativa válida y efectiva para la cirugía ambulatoria, presentando incluso algunos beneficios al compararla con la anestesia general. Los tiempos de ejecución, recuperación y alta si bien tienden a ser mayores que la anestesia general, el uso de anestésicos locales de duración corta ha mejorado estos outcomes. Por otro lado, la adición de otros beneficios (como el menor requerimiento de medicamentos de rescate) pueden posicionarla como una mejor alternativa aún.

\section{Anestesia peridural ambulatoria}

Podemos tener en consideración la anestesia peridural para algunos casos, como cirugías prolongadas o duración incierta. Algunos puntos relevantes en el contexto ambulatorio son: tiempos de recambio (mayor tiempo de ejecución de la técnica), tiempo de recuperación para el alta, micción post anestesia y dolor lumbar post punción (41). Algunas ventajas que podemos obtener de esta técnica son mayor control de cambios hemodinámicos, titulación de la dosis de anestésico local al dermatoma deseado y regulación del nivel de bloqueo motor y/o sensitivo. Sin embargo, puede aumentar el tiempo de preparación del paciente antes de poder iniciar el procedimiento quirúrgico, lo que debemos tener en cuenta en cirugía ambulatoria para lograr una buena eficiencia del uso del pabellón.

En un estudio que comparó anestesia general, peridural y espinal para artroscopía de rodilla, se observó que los tiempos de alta de las dos primeras era similar, y el alta de los pacientes con anestesia espinal era más tardía y con mayor incidencia de efectos adversos (42). La aparición de los anestésicos locales de duración corta como cloroprocaína. (43), ha demostrado que la anestesia peridural es también una buena alternativa en cirugías como la artroscopia de rodilla ambulatoria, facilitando altas más precoces en comparación a otros anestésicos locales.

Por lo tanto, la anestesia peridural es una técnica que debemos mantener dentro de nuestras opciones de anestesias ambulatorias, pero teniendo en consideración las variables del procedimiento en sí y facilitando los tiempos de recuperación mediante el uso de anestésicos locales de duración corta.

\section{Referencias}

1 Urmey W. Spinal anaesthesia for outpatient surgery. Best Practice \& Research Clinical Anaesthesiology Vol. 17, No. 3, pp. 335-346, 2003

2 Song D, Greilich N, White P, et al. Recovery profiles and costs of anesthesia for outpatient unilateral inguinal herniorrhaphy. Anesth Analg 2000;91:876-81.

3 Liu SS. A comparison of regional versus general anesthesia for ambulatory anesthesia: a meta-analysis of randomized controlled trials. Anesth Analg 2005;101: 1634-42.

4 Capdevilla et al. Factors Determining the Choice of Spinal Versus General Anesthesia in Patients Undergoing Ambulatory Surgery: Results of a Multicenter Observational Study. Adv Ther https://doi.org/10.1007/ s12325-019-01171-6 2019

5 Hutton M, Brull R, Macfarlane AJ. Regional anaesthesia and outcomes. BJA Education, 2018 (2): $52-56$

6 Gan TJ, Diemunsch P, Habib A, et al. Consensus guidelines for the management of postoperative nausea and 
vomiting. Anesth Analg 2014; 118: 85-113

7 Davis N, Lee M, Lin AY, et al. Post-operative cognitive function following general versus regional anesthesia: a systematic review. J Neurosurg Anesthesiol 2014; 26: 369-76

8 Smith LM, Cozowicz C, Uda Y, Memtsoudis SG, Barrington MJ. Neuraxial and combined neuraxial/general anesthesia compared to general anesthesia for major truncal and lower limb surgery: a systematic review and meta-analysis. Anesth Analg 2017; 125: 1931-45

9 Memtsoudis SG, Stundner O, Rasul R, et al. Sleep apnea and total joint arthroplasty under various types of anesthesia. Reg Anesth Pain Med 2013; 38: 274-81

10 Tran K, Cimon K, Severn M, et al. Aerosol generating procedures and risk of transmission of acute respiratory infections to healthcare workers: a systematic review. PLoS One 2012;7:e35797

11 Singleton MN, Soffin EM. Daring discourse: are we ready to recommend neuraxial anesthesia and peripheral nerve blocks during the COVID-19 pandemic? A pro-con. Reg Anesth Pain Med 2020;0:1-4. doi:10.1136/ rapm-2020-1016530

12 Zaric D, Christiansen C, Pace NL, et al. Transient neurologic symptoms after spinal anesthesia with lidocaine versus other local anesthetics: a systematic review of randomized, controlled trials. Anesth Analg 2005;100:1811-6.

13 Lemoine A, Mazoit JX, Bonnet F. Modelling of the optimal bupivacaine dose for spinal anaesthesia in ambulatory surgery based on data from systematic review. Eur J Anaesthesiol 2016; 33:846-852

14 Nair GS, Abrishami A, Lermitte J, Chung F. Systematic review of spinal anaesthesia using bupivacaine for ambulatory knee arthroscopy. British Journal of Anaesthesia 102 (3): 307-15 (2009)

15 Liu SS, Ware PD, Allen HW, et al. Dose-response characteristics of spinal bupivacaine in volunteers: clinical implications for ambulatory anesthesia. Anesthesiology 1996; 85: 729-36

16 Fanelli A, Ghisi D, Allegri M. Is spinal anaesthesia a suitable technique for ultra-short outpatient procedures? Acta Biomed 2013; 84:76-80

17 Goldblum E, Atchabahian A. The use of 2-chloroprocaine for spinal anaesthesia. Acta Anaesthesiol Scand 2013; 57:545-552.

18 Fanelli y Breebaart MB, Teune A, Sermeus LA, Vercauteren MP. Intrathecal chloroprocaine vs. lidocaine in day-case surgery: recovery, discharge and effect of prehydration on micturition. Acta Anaesthesiol Scand 2014; 58:206-213

19 J. Boublik et al. Prilocaine spinal anesthesia for ambulatory surgery: A review of the available studies. Anaesth Crit Care Pain Med 35 (2016) 417-421.

20 Camponovo C, Fanelli A, Ghisi D, Cristina D, Fanelli G. A prospective, doubleblinded, randomized, clinical trial comparing the efficacy of $40 \mathrm{mg}$ and $60 \mathrm{mg}$ hyperbaric $2 \%$ prilocaine versus $60 \mathrm{mg}$ plain $2 \%$ prilocaine for intrathecal anesthesia in ambulatory surgery. Anesth Analg 2010;111:568-72.

21 Gebhardt V, Herold A, Weiss C, Samakas A, Schmittner MD. Dosage finding for low-dose spinal anaesthesia using hyperbaric prilocaine in patients undergoing perianal outpatient surgery. Acta Anaesthesiol Scand 2013;57:249-56.

22 Borghi B, Stagni F, Bugamelii S et al. Unilateral Spinal Block for Outpatient Knee Arthroscopy: A DoseFinding Study. Journal of Clinical Anesthesia 15:351-356, 2003

23 Fanelli G, Borghi B, Casati A et al. Unilateral bupivacaine spinal anesthesia for outpatient knee arthroscopy. Can J Anesth 2000 / 47: 8 / pp 746-751

24 Pavlin DJ, Pavlin EG, Gunn HC, et al. Voiding in patients managed with or without ultrasound monitoring of bladder volume after outpatient surgery. Anesth Analg. 1999;89:90-97.

25 Tammela T, Kontturi M, Lukkarinen O. Postoperative urinary retention. I. Incidence and predisposing factors. Scand J Urol Nephrol. 1986;20:197-201.

26 Yogendran S, Asokumar B, Cheng DC, et al. A prospective randomized double blinded study of the effect of intravenous fluid therapy on adverse outcomes on outpatient surgery. Anesth Analg. 1995;80:682-686.

27 Mulroy M and Alley E. Management of Bladder Volumes When Using Neuraxial Anesthesia. International anesthesiology clinics. Volume 50, Number 1, 101-110. 
28 Mulroy MF, Salinas FV, Larkin KL, et al. Ambulatory surgery patients may be discharged before voiding after short-acting spinal and epidural anesthesia. Anesthesiology. 2002;97:315-319

29 Halpern S \& Preston R. Postdural puncture headache and spinal needle design. Metaanalysis. Anesthesiology 1994; 81: 1376-1383.

30 DelPizzo K, Cheng J, Dong N et al. Post-Dural Puncture Headache is Uncommon in Young Ambulatory Surgery Patients. HSSJ (2017) 13:146-151.

31 Di Cianni S, Rossi M, Casati A, Cocco C, Fanelli G. Spinal anesthesia: an evergreen technique. Acta Biomed 2008; 79: 9-17.

32 Faure E, Moreno R, Thisted R. Incidence of postdural puncture headache in morbidly obese parturients. Reg Anesth 1994; 19: 361-3.

33 Schmittner MD, Terboven T, Dluzak M, Janke A, Limmer ME, Weiss C, et al. High incidence of post-dural puncture headache in patients with spinal saddle block induced with Quincke needles for anorectal surgery: a randomised clinical trial. Int J Colorectal Dis 2010; 25: 775- 81.

34 Ahmed SV, Jayawarna C, Jude E. Post lumbar puncture headache: diagnosis and management. Postgrad Med J 2006; 82: 713-6.

35 Richman JM, Joe EM, Cohen SR, Rowlingson AJ, Michaels RK, Jeffries MA, et al. Bevel direction and postdural puncture headache: a metaanalysis. Neurologist 2006; 12: 224-8.

36 Reina M, Puigdellívol-Sánchez A, Gatt S et al. Electron Microscopy of Dural and Arachnoid Disruptions After Subarachnoid Block. Regional Anesthesia and Pain Medicine . Volume 42, Number 6, November-December 2017

37 Etezadi F, Karimi Yarandi K, Ahangary A, et al. The effect of needle type, duration of surgery and position of the patient on the risk of transient neurologic symptoms. Anesth Pain Med 2013; 2:154-158.

38 Forget P, Borovac JA, Thackeray EM, Pace NL. Transient neurological symptoms (TNS) following spinal anaesthesia with lidocaine versus other local anaesthetics in adult surgical patients: a network meta-analysis. Cochrane Database of Systematic Reviews 2019, Issue 12. Art. No.: CD003006.

39 Vaghadia H, Viskari D, Mitchell G et al. Selective spinal anesthesia for outpatient laparoscopy. I: Characteristics of three hypobaric solutions. Can J Anesth 2001 / 48: 3 / pp 256-260

40 De Santiago J, Santos-Yglesias J, Giron J, et al. Low-Dose $3 \mathrm{mg}$ Levobupivacaine Plus $10 \mathrm{~g}$ Fentanyl Selective Spinal Anesthesia for Gynecological Outpatient Laparoscopy. Anesthesia \& Analgesia Vol. 109, No. 5, November 2009

41 Alley E and Mulory M. Neuraxial Anesthesia for Outpatients. Anesthesiology Clin 32 (2014) 357-369

42 Mulroy MF, Larkin KL, Hodgson PS, et al. A comparison of spinal, epidural, and general anesthesia for outpatient knee arthroscopy. Anesth Analg 2000;91:860-4.

43 Yang Z, Dezhan L, Zhang K et al. Comparison of epidural anesthesia with chloroprocaine and lidocaine for outpatient knee arthroscopy. Journal of Orthopaedic Surgery 27(3) 1-4. 2019 
CARSACH

página 54 